\title{
OTIMIZAÇÃO DE RECURSOS PARA A OPERAÇÃO DE INSTALAÇÃO DE ÂNCORAS DE EQUIPAMENTOS OFFSHORE
}

\author{
Oscar Brito Augusto * \\ Bernardo Luís Rodrigues Andrade \\ Departamento de Engenharia Naval e Oceânica \\ Escola Politécnica - Universidade de São Paulo \\ São Paulo - SP \\ E-mails: oscar.augusto@poli.usp.br \\ beluroan@usp.br \\ Ronaldo Rosa Rossi \\ Centro de Pesquisas \\ Petrobrás \\ Rio de Janeiro - RJ \\ E-mail: ronaldorr@cenpes.petrobras.com.br \\ * Corresponding author/autor para quem as correspondências devem ser encaminhadas \\ Recebido em 05/2001, aceito em 12/2002 após 1 revisão
}

\section{Resumo}

Neste trabalho é apresentada uma metodologia para o planejamento da operação de instalação de âncoras de equipamentos offshore, navios e plataformas, visando a otimização dos recursos necessários, embarcações de apoio e cabos de trabalho, por meio da minimização de uma função objetivo com base em múltiplos critérios. Como ganho adicional, inerente à metodologia proposta, consegue-se a automação do processo de planejamento de instalação, que nos moldes tradicionais é feito na base da tentativa e erro, onde o planejador, utilizando algum aplicativo para o cálculo de ancoragem, decide quanto de recursos deve ser aplicado ao problema tentando fazer com que a âncora atinja o alvo prédefinido no projeto do sistema de ancoragem.

Palavras-chave: ancoragem, plataformas de petróleo, otimização, múltiplos critérios.

\begin{abstract}
In this work is explored a planning methodology for deep water anchor lines deployment in offshore platforms and floating production systems aiming operational resources optimization, by minimizing a multi criteria objective function. As an additional advantage, inherited from the proposed methodology, the planning automation is achieved. The planning automation overcomes the traditional way to do in a trial error basis, where an engineer, using a anchoring software, decides how much of work wire and anchoring line must be paid out from both the floating system and the supply vessel and additionally which horizontal force must be applied to the line trying settle the anchor on a previously defined target in the ocean floor.
\end{abstract}

Keywords: anchorage, oil platforms, optimization, multiple criteria. 


\section{Introdução}

Uma operação muito comum para os grupos que ancoram e desancoram plataformas de perfuração bem como outros equipamentos ligados à exploração de petróleo no mar consiste na instalação das âncoras e das linhas de ancoragem.

Embora esta operação ocorra com muita freqüência, pouca atenção se tem dado ao desenvolvimento de simuladores e aplicativos que auxiliem os engenheiros no cálculo das etapas de lançamento e dos recursos a serem utilizados para tal operação. O processo consiste em um rebocador ou embarcação de apoio içar, através da âncora, a linha de ancoragem da plataforma ou navio, por intermédio de um cabo de apoio, work wire, e durante o içamento, os guinchos, tanto do lado da plataforma quanto do lado da embarcação de apoio, liberam quantidades restritas das linhas até que a âncora atinja o leito marinho, segundo um planejamento prévio de quantas etapas devem ser executadas e o quanto de linha deve ser liberada em cada etapa, conforme se mostra na figura 1.

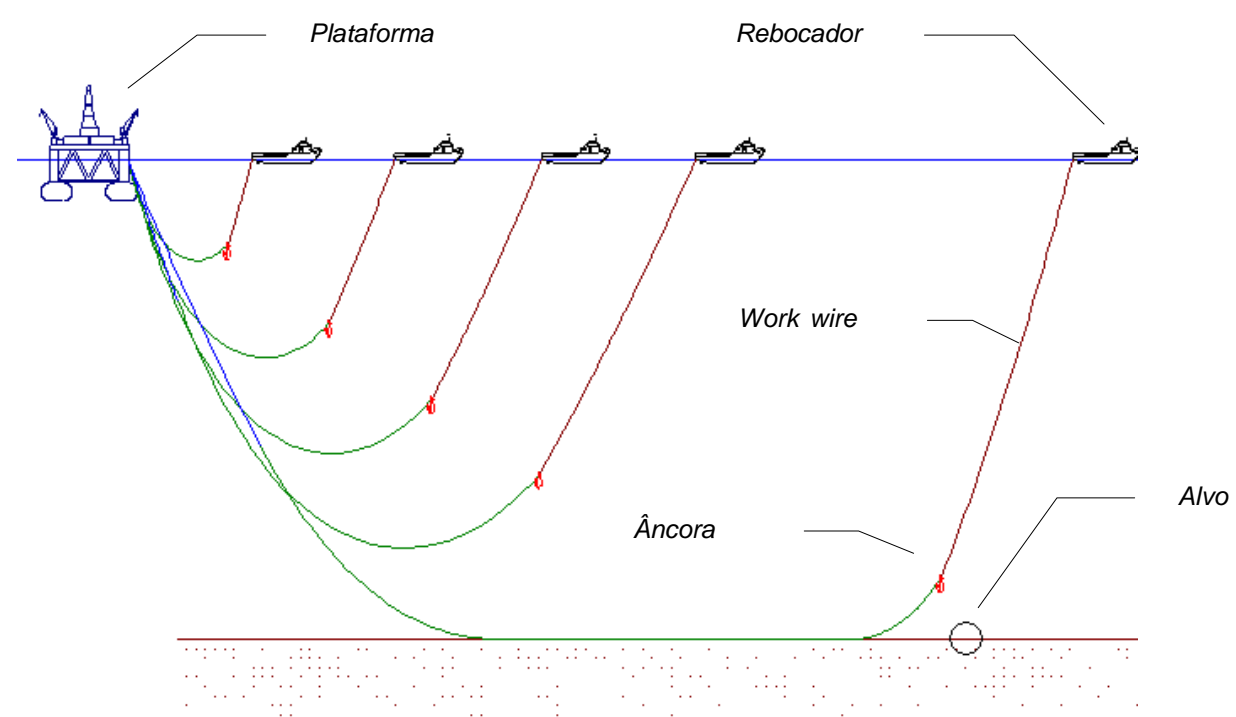

Figura 1 - Simulação da operação de lançamento de âncora

\section{Nomenclatura}

$\mathbf{X} \quad$ vetor de variáveis de projeto

$f_{j}(\mathbf{X}) \quad$ j-ésima função objetivo

$g_{j}(\mathbf{X})$ j-ésima função de restrição interior

$h_{j}(\mathbf{X})$ j-ésima função de restrição homogênea

$b_{j} \quad$ meta para a j-ésima função objetivo

$d_{j}^{ \pm} \quad$ desvio da j-ésima função objetivo

$r \quad$ distância fair lead a um ponto da linha

z profundidade do ponto na catenária

$w_{i} \quad$ peso linear de um segmento de linha

$G_{i} \quad$ força concentrada no i-ésimo segmento

$p_{\text {ref }}$ ponto de referência do fundo

$w_{j}^{ \pm} \quad$ peso associado ao desvio da j-ésima meta

$p_{j} \quad$ prioridade da j-ésima função objetivo

$\alpha$ ângulo de inclinação do fundo

$\psi \quad$ função de mérito de múltiplos critérios

$V$ componentes verticais de força seg

TDP ponto de toque da linha no fundo

$E A$ rigidez axial de um segmento homogêneo

$\theta \quad$ âng. de inclinação num ponto da linha

L comprimento (work wire, segmento, etc) 
Atualmente, para o planejamento destas etapas, se dispõe de sistemas para cálculo de linhas em catenária, onde o técnico responsável pela tarefa deve decidir o quanto deve lançar de linha em cada uma das embarcações envolvidas na operação e qual deve ser a distância entre elas, na tentativa de fazer com que a âncora caminhe para o alvo desejado. Se não, deve-se tentar novos valores até que se atinja o objetivo. Tal procedimento é pouco produtivo e, embora possa se atingir a condição desejada, nada é garantido quanto a adequabilidade da solução encontrada em relação aos recursos necessários para efetuar, de fato, a operação de lançamento.

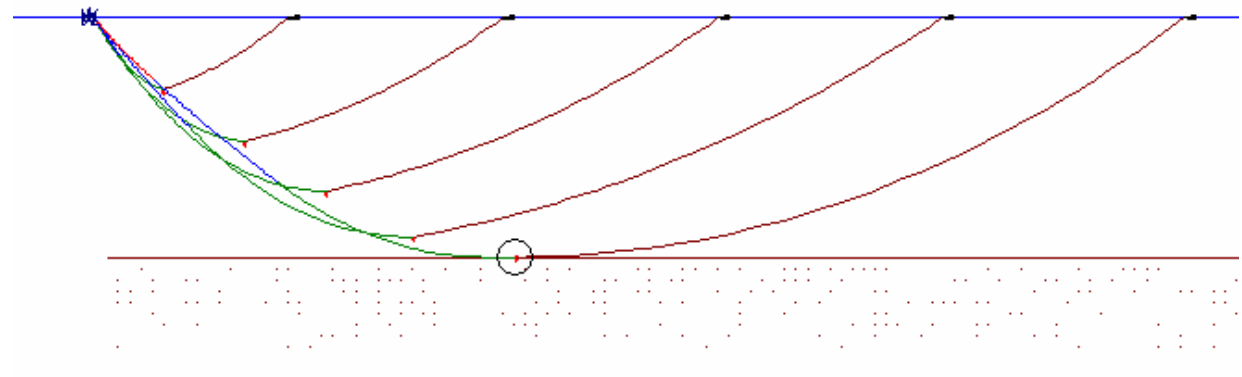

Figura 2 - Simulação - Âncora atinge o alvo Força Horizontal 79.86 tons Work wire: $2679.9 \mathrm{~m}$

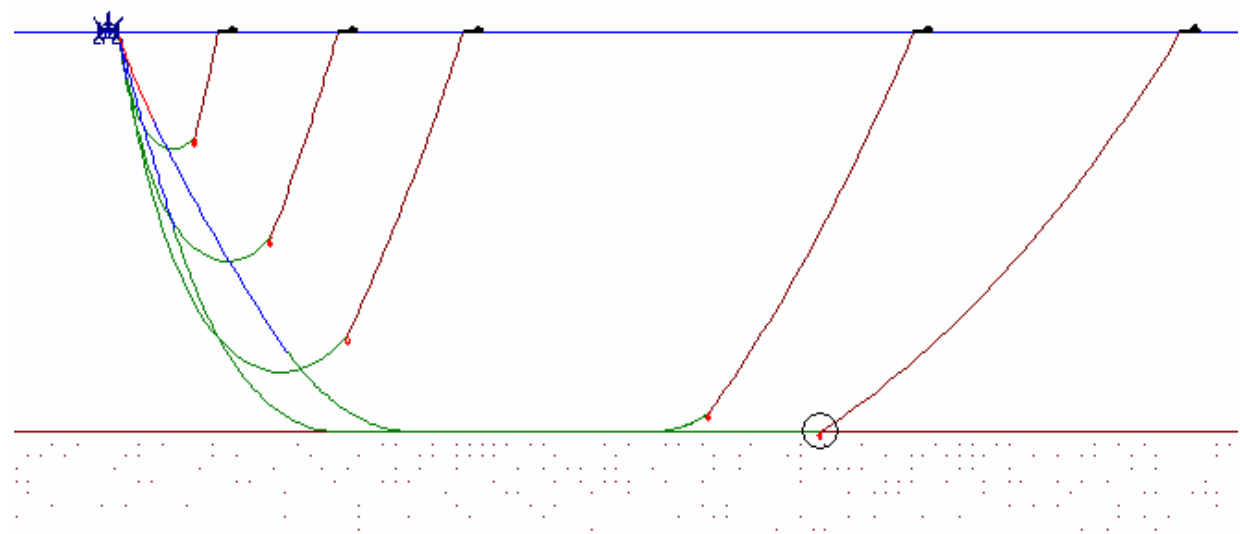

Figura 3 - Simulação - Âncora atinge o alvo Força Horizontal 26.62 tons Work wire: $1761.6 \mathrm{~m}$

Nas figuras 1, 2, 3 e 4 encontram-se situações distintas para a mesma operação de lançamento de uma âncora. Na primeira o alvo não foi atingido. Nas demais o alvo pretendido foi atingido, porém as características finais diferem substancialmente entre si, conforme se verifica na tabela T1. 


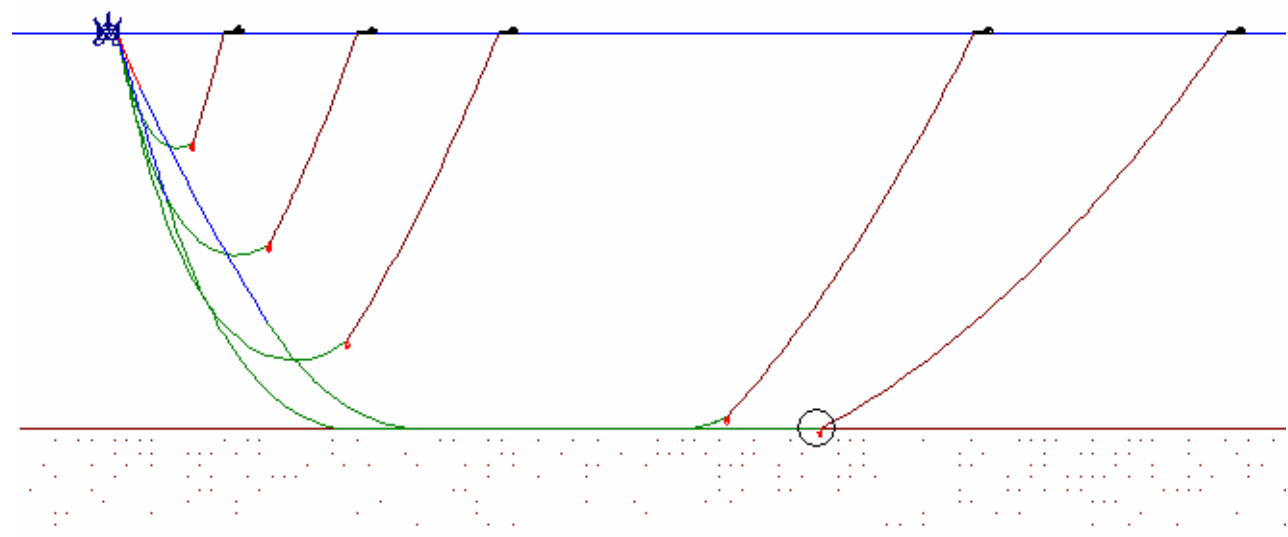

Figura 4 - Simulação - Âncora atinge o alvo Força Horizontal 30.62 tons Work wire: $1285.7 \mathrm{~m}$

Tabela T1 - Desempenho de procedimentos de lançamento de âncora.

\begin{tabular}{|c|c|r|r|r|r|r|}
\hline \multirow{2}{*}{$\begin{array}{c}\text { Caso } \\
\text { Fig. }\end{array}$} & \multicolumn{2}{|c|}{ Plataforma } & \multicolumn{2}{c|}{ Rebocador } & \multicolumn{2}{c|}{ Distância } \\
\cline { 2 - 7 } & Comprim & Força H & Comprim & Força H & Plat-Reboc & FLead-Anc \\
\hline 1 & 1862.0 & 16.95 & 1071.3 & 1.89 & 1702.8 & 1337.7 \\
\hline 2 & 1860.5 & 121.28 & 2679.9 & 79.86 & 4068.4 & 1571.0 \\
\hline 3 & 1761.6 & 26.62 & 1209.3 & 26.62 & 2373.7 & 1569.8 \\
\hline 4 & 1682.9 & 30.62 & 1285.7 & 30.62 & 2490.7 & 1579.0 \\
\hline
\end{tabular}

Retendo-se apenas as simulações onde o alvo é atingido, verifica-se, na figura 2 que, para atingir o alvo, o rebocador deve lançar $2679.9 \mathrm{~m}$ de work wire e impor uma força horizontal de 79.86 t. Na situação mostrada na figura 3 ele deve lançar $1209.3 \mathrm{~m}$ de work wire e impor uma força horizontal de 26.62 t. Finalmente, na figura 4, o alvo é atingido com o rebocador lançando $1285.7 \mathrm{~m}$ e exercendo uma força horizontal da ordem de $30.62 \mathrm{t}$ no work wire.

Fica patente que além de ser um procedimento dispendioso, pois exige do técnico um difícil exercício iterativo de tirocínio, é duvidoso quanto à possibilidade de um sucesso na simulação e, mesmo nos casos em que o alvo é atingido, não se garante a otimização dos recursos necessários para a operação de lançamento, destacando-se a minimização da força horizontal na linha, o que possibilita a alocação de um rebocador com menor bolard pull ou mesmo em outras situações, onde seriam alocados dois rebocadores, por limitações de força disponível, se utilize apenas um.

Com a produção de petróleo brasileiro em águas cada vez mais profundas, a necessidade de se alocar ou rebocadores com maior bollard pull, ou mesmo mais de um rebocador, que unidos executam uma operação, tende a se tornar freqüente. Nestas condições se faz premente uma ferramenta que além de automatizar o processo de cálculo das etapas de lançamento também procure as soluções que minimizem os recursos necessários para a instalação das âncoras. 
Notando que o custo operacional de um rebocador com bollard pull de $50000 \mathrm{t}$ gira em torno de US\$ 6000.00 por dia; supondo que uma operação de ancoragem e desancoragem dure de 7 a 15 dias; supondo que na situação de instalação de uma âncora onde seria necessário apenas um rebocador para a operação e, por uma situação de cálculo não otimizado, estivessem sendo alocados dois, o desperdício financeiro em custo se daria na ordem de US\$ 90 000,00. Admitindo-se que umas doze operações não otimizadas como as mencionadas ocorram durante o espaço de um ano, o desperdício atingiria cifras na ordem de US\$ 1000 000,00 ao ano.

Alem do mais, a automatização do processo geraria outras receitas indiretas, como um menor tempo dos técnicos na execução desta tarefa de cálculo e, conseqüentemente, maior produtividade no setor de operações de ancoragem.

\section{Otimização por múltiplos critérios}

O problema descrito na introdução pode ser facilmente resolvido através da técnica denominada de otimização por múltiplos critérios (goal programming), que pode ser considerada como uma classe particular de um problema mais geral de programação matemática.

Sejam:

$$
\boldsymbol{X}=\left(x_{1}, x_{2}, x_{3}, \ldots x_{n}\right)^{T}
$$

um vetor de $n$ variáveis independentes;

$$
f(X)=f\left(x_{1}, x_{2}, x_{3}, \ldots x_{n}\right)^{T}
$$

a função a ser otimizada;

$$
g_{j}(X) \geq 0
$$

para $\mathrm{j}=1,2, \ldots n_{g}$.as funções de restrições de fronteira; e

$$
h_{j}(X)=0
$$

para $\mathrm{j}=1,2, \ldots n_{h}$. as funções de restrições homogêneas.

Neste trabalho, a representação matemática das metas ou objetivos será descrita por:

$$
\frac{f_{j}(\mathbf{x})}{b_{j}}+d_{j}^{-}-d_{j}^{+}=1
$$

onde:

$$
\begin{aligned}
f_{j}(\mathbf{X})= & \text { meta da j-ésima função objetivo, função das variáveis de projeto } \mathbf{X} \text {, com nível } \\
& \text { de aspiração } b_{j} \\
d_{j}^{-}, d_{j}^{+}= & \text {variáveis de desvio, representando respectivamente sub ou sobre atendimento } \\
& \text { do nível de aspiração } b_{j}, \text { e com valores sempre } \geq 0 .
\end{aligned}
$$


Assim, o problema de otimização passa a ter a seguinte constituição:

$$
\begin{aligned}
& \text { se } f_{j}(\mathbf{X}) \text { precisa ser } \geq b_{j} \text {, então } d_{j}^{-} \text {é minimizado; } \\
& \text { se } f_{j}(\mathbf{X}) \text { precisa ser } \leq b_{j} \text {, então } d_{j}^{+} \text {é minimizado; } \\
& \text { se } f_{j}(\mathbf{X}) \text { precisa ser }=b_{j} \text {, então ambos } d_{j}^{-} \text {e } d_{j}^{+} \text {são minimizados; } \\
& d_{j}^{-} \cdot d_{j}^{+}=0 \text {, não pode haver simultaneamente sub e sobre atendimento da meta. }
\end{aligned}
$$

Considerando agora que com esta nova formulação as metas e objetivos passam a definir um subespaço de aspiração que deve ser atingido tanto quanto possível e que, as restrições definem um subespaço dentro do qual as soluções devem necessariamente estar inseridas para satisfazer requisitos de viabilidade, o problema de otimização pode ser expresso como um problema generalizado de programação por objetivos no qual a soma ponderada das variáveis de desvio deve ser minimizada, considerando a presença de metas e restrições. A representação matemática deste problema é a seguinte:

minimizar

$$
f=\sum_{j=1}^{N} p_{j} \cdot\left(w_{j}^{-} \cdot d_{j}^{-}+w_{j}^{+} \cdot d_{j}^{+}\right)
$$

sujeito a:

$$
\begin{aligned}
& \frac{f_{j}(\mathbf{X})}{b_{j}}+d_{j}^{-}-d_{j}^{+}=1, j=1,2, \ldots, n_{f} \\
& g_{i}(\mathbf{X}) \geq 0, \quad i=1,2, \ldots, n_{g} \\
& h_{i}(\mathbf{X})=0, \quad i=1,2, \ldots, n_{h}
\end{aligned}
$$

onde:

$w_{j}^{-}, w_{j}^{+}=$pesos associados aos desvios da j-ésima meta;

$p_{j}=$ prioridade associada à j-ésima meta.

O problema de projeto passa a ser então o de encontrar o vetor $\mathbf{X}$ das variáveis de decisão tal que as restrições, $g_{i}(\mathbf{X}) \geq 0$ e $h_{i}(\mathbf{X})=0$, sejam satisfeitas e as metas, ou objetivos, $f_{j}(\mathbf{X})$ alcançados, dentro da melhor aproximação possível. As restrições devem, evidentemente, ser consistentes, pois, de outro modo, não haverá solução para o problema. As metas, no entanto, não precisam ser e, em geral, não serão consistentes.

\section{Solução do problema de catenária com múltiplos segmentos e com as duas extremidades na superfície}

Para a modelagem do problema físico da linha, com uma extremidade fixa na embarcação a ser ancorada e a outra, na embarcação de apoio para o lançamento da âncora, utilizou-se a metodologia proposta por Oppenheim (1982), onde, para cada segmento de material homogêneo integrante da linha de ancoragem pode-se admitir a força gravitacional como sendo a única força de campo presente no sistema, e desta forma, a equação clássica da catenária para um segmento homogêneo pode ser utilizada. 




Figura 5 - Variáveis na linha

Sendo:

- $\quad w_{i}$ peso unitário na água para o i-ésimo segmento homogêneo de linha $\left(w_{i}>0\right.$ para segmentos com flutuação);

- $G_{i}$, peso concentrado suspenso na extremidade superior do i-ésimo segmento, (negativo, representa uma bóia submersa; positivo, uma poita);

- $L_{i}$, o comprimento do i-ésimo segmento homogêneo da linha;

- $f\left(r, p_{\text {ref }}, \alpha\right)$, a função que descreve a leito oceânico no plano da linha, suposto uma reta com inclinação $\alpha$.

O problema do equilíbrio de um segmento de catenária da forma acima proposta possui solução clássica a qual passamos a detalhar.

\section{O modelo analítico de uma catenária homogênea}

Na figura 6 mostra-se o modelo de um cabo suspenso onde a única força distribuída presente ao longo do comprimento do cabo e a força peso.

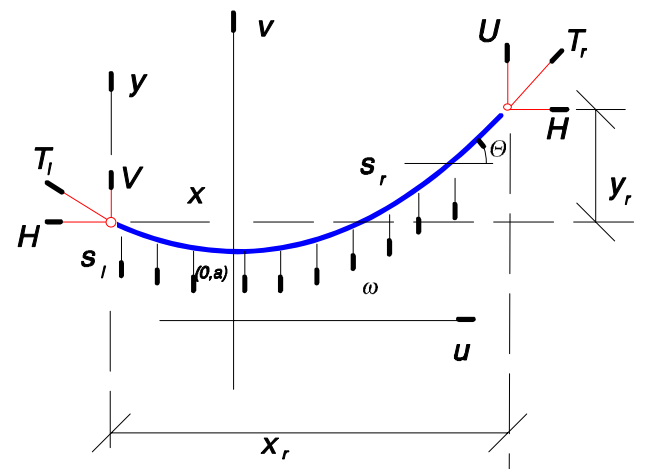

Figura 6 - O modelo de catenária ideal 
No mar estão presentes as forças de correnteza que além de contribuírem para uma componente ortogonal a ação gravitacional ou de empuxo, podem agir no sentido de retirarem o cabo de seu plano vertical. Tal limitação, embora possa parecer conflitante com as aplicações na indústria offshore, tem sido muito usada, pois permite o cálculo analítico das linhas de ancoragem, dentro das faixas de velocidades de correnteza encontradas na costa brasileira e dos materiais empregados para linhas de ancoragem.

Para incluir tais efeitos torna-se necessária a utilização de modelos mais sofisticados, tornando o modelo do problema passível de solução através da aplicação de métodos numéricos somente. O mais comum entre eles e o Método dos Elementos Finitos, onde o cabo é modelado por elementos de viga ou de treliça.

Em aplicações offshore, geralmente utilizam-se duas configurações de ancoragem:

1. convencional, com a linha em catenária, onde são empregadas as âncoras de arrasto, de menor custo;

2. taut leg, ou linha retesada, onde se utilizam as âncoras que suportam cargas verticais, de maior custo.

Adota-se uma ou outra solução dependendo das condições de fundo. Se congestionado com equipamentos há necessidade de se adotar um sistema com um curto raio de instalação das âncoras.

Na configuração em catenária, a tração no topo da linha se dá pela quantidade de linha que está suspensa do leito marinho. A modelagem de um sistema como este utilizando o Método dos Elementos Finitos só é possível com o uso de não linearidades geométricas uma vez que tal sistema se mostrará como um mecanismo. Basta imaginar uma corda, ideal frouxa, com uma extremidade fixa e a outra podendo se movimentar em um plano horizontal. A rigidez elástica inicial inexiste e, portanto, o problema, não seria resolvido pelo método dos elementos finitos linear. Por outro lado, admitindo-se que o peso ira gerar tração ao longo da linha, pode-se então, utilizar esta tração para compor a rigidez elástica inicial com a rigidez geométrica, que depende da configuração de equilíbrio, para a solução do problema.



Figura 7 - Comparação entre ancoragem convencional e taut leg 
Tal abordagem permite o uso de forças de arrasto, devido a correnteza, no cálculo do equilíbrio do cabo, porém para quase totalidade das instalações de ancoragem, a força de arrasto gerada pelas correntezas pouco distorce a configuração da linha gerada apenas pelo peso próprio, levando a conclusão de que o modelo clássico de cálculo de catenária pode ser empregado sem maiores desvios pelo efeito das forças de correnteza. Diferentemente do modelo em elementos finitos onde a não linearidade força a busca de solução de forma iterativa, o modelo clássico possui solução analítica, o que o torna propício na utilização e métodos de otimização onde muitas soluções serão pesquisadas antes de se atingir uma solução considerada ótima.

O modelo que se adotará neste trabalho é o clássico, onde cada segmento é tratado como um segmento de catenária, estando os pontos de união entre os segmentos, em equilíbrio de esforços. Desta forma, partindo-se de uma extremidade da linha, se atinge a outra, simplesmente, calculando-se as forças que se transferem de segmento para segmento. Agindo desta forma, a configuração de equilíbrio também é encontrada e caso as extremidades não coincidam com pontos a priori desejados, instaura-se um processo iterativo, com uma variável de controle, ângulo no topo, por exemplo, até que se atinja a configuração geométrica final, coerente com as condições de ancoragem.

Considere um segmento de linha elástico, conforme o mostrado na figura 6. Sendo $w>0$ o seu peso por unidade de comprimento e o par $s_{l}$ e $s_{r}$, os comprimentos entre as extremidades e o ponto inferior da linha, conforme ilustrado. As forças agindo nas extremidades dos segmentos são a tensão horizontal $H$, constante em todos os pontos do segmento e as tensões verticais $L$ e $U$, conforme o mostrado.

O equilíbrio de forças para o comprimento $s_{r} \mathrm{e}$

$$
\begin{aligned}
& T_{r} \sin \theta=w S_{r} \\
& T_{r} \cos \theta=H
\end{aligned}
$$

Seja $a=\frac{H}{w}$

Então as equações (10) podem ser reescritas como:

$$
\begin{aligned}
& \sin \theta=\frac{w s_{r}}{T_{r}}=\frac{w s_{r}}{\sqrt{H^{2}+\left(w s_{r}\right)^{2}}}=\frac{s_{r}}{\sqrt{s_{r}^{2}+a^{2}}} \\
& \cos \theta=\frac{H}{T_{r}}=\frac{a}{\sqrt{s_{r}^{2}+a^{2}}}
\end{aligned}
$$

Mas

$$
\begin{aligned}
& \sin \theta=\frac{d v}{d s}=\frac{s_{r}}{\sqrt{s_{r}{ }^{2}+a^{2}}} \\
& \cos \theta=\frac{d u}{d s}=\frac{a}{\sqrt{s_{r}{ }^{2}+a^{2}}}
\end{aligned}
$$


Se este procedimento for repetido para a porção $s_{l}$ do segmento e as equações para ambos os lados forem combinadas, as coordenadas, horizontal e vertical, x e y respectivamente, serão escritas na forma integral:

$$
\begin{aligned}
& x=u_{r}-u_{l}=\int_{0}^{s_{r}} \frac{a d s}{\sqrt{s^{2}+a^{2}}}-\int_{0}^{s_{l}} \frac{a d s}{\sqrt{s^{2}+a^{2}}} \\
& y=v_{r}-v_{l}=\left[a+\int_{0}^{s_{r}} \frac{a d s}{\sqrt{s^{2}+a^{2}}}\right]-\left[a+\int_{0}^{s_{l}} \frac{a d s}{\sqrt{s^{2}+a^{2}}}\right]
\end{aligned}
$$

Após a integração tais coordenadas podem ser reescritas:

$$
\begin{aligned}
& x=a \ln \frac{a+\sqrt{s_{r}^{2}+a^{2}}}{a+\sqrt{s_{l}^{2}+a^{2}}}=\frac{H}{w} \ln \frac{s_{r} w+\sqrt{\left(s_{r} w\right)^{2}+H^{2}}}{s_{l} w+\sqrt{\left(s_{l} w\right)^{2}+H^{2}}} \\
& y=\sqrt{s_{r}^{2}+a^{2}}-\sqrt{s_{l}^{2}+a^{2}}=\frac{1}{w} a \ln \frac{a+\sqrt{s_{r}^{2}+a^{2}}}{=}=\frac{H}{w}\left[\sqrt{\left(s_{r} w\right)^{2}+H^{2}}-\sqrt{\left(s_{l} w\right)^{2}+H^{2}}\right]
\end{aligned}
$$

As equações (14) podem ser escritas em termos das forças verticais ao invés das variáveis $s_{l} w$ e $s_{r} w$.

$$
\begin{aligned}
& x=\frac{H}{w} \ln \frac{U+\sqrt{U^{2}+H^{2}}}{L+\sqrt{L^{2}+H^{2}}} \\
& y=\frac{1}{w}\left[\sqrt{U^{2}+H^{2}}-\sqrt{L^{2}+H^{2}}\right]
\end{aligned}
$$

onde

$$
\begin{aligned}
& L=s_{l} w \\
& U=s_{r} w
\end{aligned}
$$

As equações (15) e (16) são gerais. Elas são válidas para $w<0$ ou $w>0$.

\section{Linha com diversos segmentos}

Para uma linha composta por diversos segmentos, o algoritmo proposto para determinar-se a configuração da linha é estabelecido de forma iterativa. Admitindo-se conhecida a tensão no topo da linha, $T_{r}$ os seguintes passos são seguidos:

1. Admite-se o ângulo de inclinação da linha no topo, $\theta_{0}$, junto à extremidade que pertence à embarcação a ser ancorada e, com isso, a projeção vertical de $T_{r}$, na extremidade superior, $U$, como

$$
\begin{aligned}
& U=T_{r} \cos \left(\theta_{0}\right) \\
& H_{p}=T_{r} \sin \left(\theta_{0}\right)
\end{aligned}
$$


2. A partir do topo resolve-se o problema de um segmento de catenária homogênea, calculando-se a geometria e a força na extremidade inferior do segmento, $V_{i}$,

$$
V_{i}=g_{i}\left(G_{i}, r_{i}, L_{p}, w_{p}, E A_{p}\right)+L_{i} w_{i}-U_{i}
$$

onde:

$g_{i} \quad$ é a força concentrada na extremidade superior do segmento, gerada pela bóia ou poita, função da imersão da bóia, caso esta não esteja complemente submersa, ou a força concentrada gerada pela poita, caso esta esteja em contato com o fundo.

$L_{p}$ é o comprimento do pendente, admitido de segmento homogêneo único, com peso submerso $w_{p}$ e rigidez axial $E A_{p}$.

$r_{i} \quad$ é a abscissa da extremidade superior do i-ésimo segmento.

Para uma bóia cilíndrica, de volume $V_{T}$, imersa parcialmente, com calado (o calado da bóia dependerá da rigidez axial do pendente, portanto, localmente, tem-se um processo iterativo para definir, a partir do ponto de conexão do pendente da linha, qual deverá ser a o calado da bóia, sua força de empuxo, a deformação do pendente, que por sua vez resulta em um novo calado para a bóia; um método de aproximações sucessivas garante uma rápida convergência neste processo), $h$, tem-se

$$
g_{i}=\frac{\pi D^{2} h}{4 V_{T}} G_{i}
$$

3. Pelo equilíbrio do ponto da extremidade inferior do segmento, calcula-se a projeção da força vertical, $U_{i+1}$, atuante na extremidade superior do próximo segmento; e assim sucessivamente até o último segmento.

4. Calcula-se a distância vertical da extremidade oposta da linha - a que pertence à embarcação de apoio - à superfície oceânica. Como esta extremidade deve estar na superfície, a função distância deve ser identicamente nula.

$$
\delta_{E}=z_{E}
$$

5. Através de um método iterativo, Newton por exemplo (por se tratar de buscar o zero de uma função, alternativamente pode-se utilizar qualquer algoritmo de otimização para a solução do problema.), ajusta-se o ângulo no topo, $\theta_{0}$, até que a função anteriormente descrita se anule, ou seja minimiza-se a função.

$$
\delta_{E}=\left|z_{E}\right|
$$

6. Neste ponto, verifica-se se algum ponto da linha atinge o solo oceânico.

7. Caso não ocorra o toque, a configuração da linha está determinada.

8. Caso contrário, inicia-se um outro procedimento onde as etapas 1, 2 e 3 são as mesmas anteriormente definidas.

9. Como, à priori já se sabe que a linha toca o solo, procura-se, para um dado ângulo de topo $\theta_{0}$, o ponto da linha cuja tangente é idêntica a inclinação do fundo. Tal ponto é candidato ao ponto de contato da linha com fundo, o TDP (Touch Down Point). 
10. Verifica-se a distância deste ponto ao fundo. Esta passa a ser a nova função distância para a qual procura-se, iterativamente o angulo $\theta_{0}$ de tal forma que ela se anule.

$$
\delta_{T D P}=z_{T D P}-f\left(r_{T D P}, p_{r e f}, \alpha\right)
$$

11. Encontrado o ângulo $\theta_{0}$ que anula esta função, dá-se início a um novo processo iterativo onde, a geometria da catenária suspensa no lado da embarcação a ser ancorada já está definida, desde o fair lead até o TDP. Então arbitra-se uma porção do comprimento da linha que repousa do fundo, $L_{f}$, a partir do TDP, definindo-se o segundo $\mathrm{TDP}_{2}$.

12. A partir do segundo TDP, $\mathrm{TDP}_{2}$, monta-se a geometria de catenária suspensa até a extremidade da embarcação de apoio.

13. Calcula-se a distância vertical da extremidade oposta da linha - a que pertence à embarcação de apoio - à superfície oceânica. Como esta extremidade deve estar na superfície, a função distância deve ser identicamente nula.

$$
\delta_{E}=\left|z_{E}\right|
$$

14. Através de um método iterativo, Newton por exemplo, ajusta-se o comprimento no fundo, $L_{f}$, até que a função anteriormente descrita, equação 17 , se anule.

Em vários pontos do algoritmo para o cálculo da catenária foi sugerido um método iterativo para o cálculo de uma raiz de uma função erro. Vários métodos podem ser utilizados para tal. No trabalho foi utilizado o Método de Newton, que possibilita a convergência do processo em poucas iterações, embora, nos casos mencionados, as derivadas das funções erro tivessem que ser obtidas por diferenças finitas.

\section{Otimização por múltiplos objetivos para o problema de lançamento}

Para a situação de lançamento, podemos definir como variáveis de decisão:

a) o comprimento de work wire, $L_{w w}$, a ser lançado pela embarcação de apoio;

b) o comprimento de linha, $L_{p}$, a ser lançado pela embarcação a ser ancorada; e

c) a força horizontal, $H_{p}$, a ser exercida pela embarcação a ser ancorada.

Uma vez definidas estas variáveis, pode-se encontrar a geometria da linha em catenária que satisfaz às condições estabelecidas. Nesta configuração a âncora atinge a posição $P_{A n c}=P_{A n c}\left(r_{A n c}, z_{A n c}\right)$, no fundo ou não, da qual podemos definir a função distância, $\delta_{a}$, entre o alvo, $P_{A l v o}=P_{\text {Alvo }}\left(r_{A l v o}, Z_{\text {Alvo }}\right)$, e a âncora,

$$
\delta_{a}=\operatorname{dist}\left(P_{\hat{A} n c}\left(H_{P}, L_{w w}, L_{P}\right), P_{\text {Alvo }}\right)
$$

a força horizontal na embarcação de apoio,

$$
H_{R}=H_{R}\left(H_{E}, L_{w w}, L_{L}\right)
$$


Como objetivos a serem minimizados devemos ter:

$$
\begin{aligned}
& H_{R}+d_{H}^{-}-d_{H}^{+}=H_{R} \min \\
& L_{w w}+d_{L w}^{-}-d_{L w}^{+}=L_{w w} \min \\
& L_{P}+d_{P}^{-}-d_{P}^{+}=L_{P} \min \\
& \delta_{a}+d_{a}^{-}=0
\end{aligned}
$$

Note que, a força horizontal a ser aplicada na linha, no lado da embarcação de apoio, pode ser diferente da força horizontal aplicada no lado da embarcação a ser ancorada se a linha, composta pelo work wire e linha de ancoragem encostar-se ao solo oceânico e o atrito entre ela e o solo não for nulo:

Com isto, obtém-se a função objetivo a ser minimizada:

$$
\min \left(d_{H}^{-}+d_{H}^{+}+d_{L w}^{-}+d_{L w}^{+}+d_{P}^{-}+d_{P}^{+}+d_{a}^{-}\right)
$$

Como se pretende minimizar $H_{r}, L_{w w}$ e $L_{P}$, adota-se como alvo os valores:

$$
H_{R} \min =0, L_{w w} \min =0, \quad L_{P} \min =0
$$

Para a solução do problema de minimização com múltiplos objetivos utilizou-se o método direto de otimização, Augusto (1998), acreditando-se que para o problema de ancoragem, mesmo com um pequeno número de variáveis, as funções derivadas, sejam de tensões, sejam de deslocamentos, não são trivialmente obtidas, sendo necessário, portanto, o seu cálculo numericamente processado por algum algoritmo de diferenças finitas, o que, de per si, prejudicaria o desempenho dos métodos indiretos de otimização.

\section{Resultados}

Como aplicação da metodologia proposta tome-se como exemplo a operação de lançamento de uma linha de ancoragem de um navio FPSO (Floating Production, Storage and OffLoading) locada em $200 \mathrm{~m}$ de lâmina d'água, em um talude continental com inclinação de $5^{\circ}$, com a linha composta de 2 segmentos de amarra, com as propriedades mostradas na tabela T2. Pelo projeto do sistema de ancoragem, tal linha deve ter sua âncora cravada a um raio de ancoragem de $622 \mathrm{~m}$, no azimute de $142^{\circ}$, relativo ao norte geográfico. Para a operação de lançamento, acrescenta-se a este valor uma margem operacional de $50 \mathrm{~m}$ para que ao ser içada a linha a âncora arraste, penetre e unhe no solo na posição desejada. Desta forma, o alvo para o lançamento, o raio de ancoragem para a operação de lançamento se modifica para 672 m, com uma tolerância de $1 \mathrm{~m}$. Dispõe-se de embarcações de apoio com bollard pull máximo de 50 t e 1000 m de work wire. Pode-se verificar, através dos resultados mostrados na Tabela T3, que os objetivos são plenamente atingidos. 


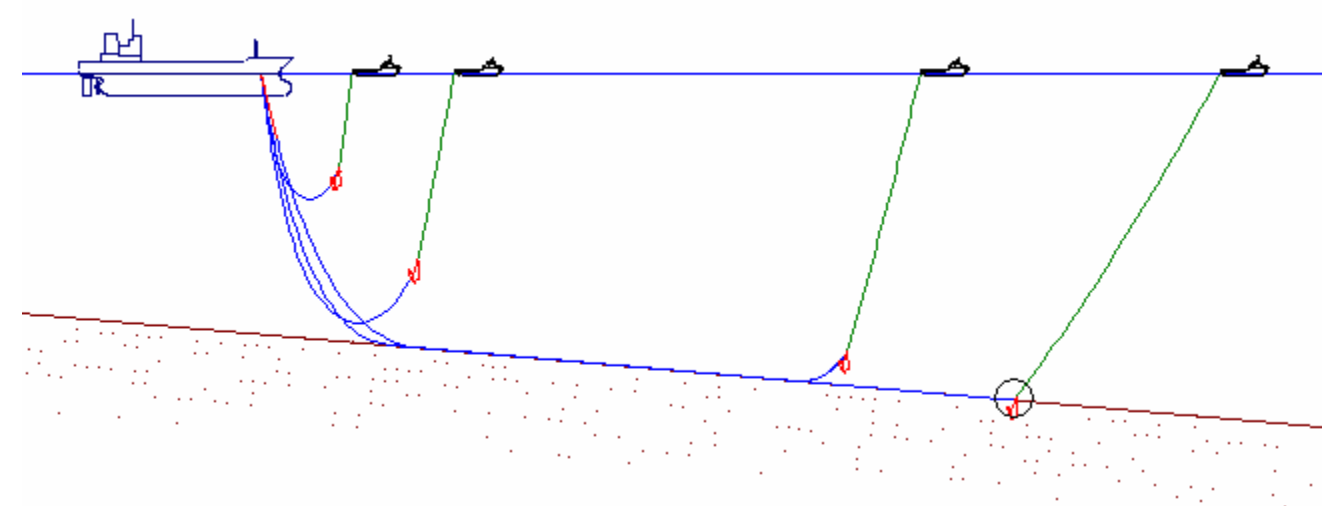

Figura 8 - A operação de lançamento

Tabela T2 - Propriedades da linha a ser instalada

\begin{tabular}{|r|c|r|r|r|r|r|r|}
\hline Seg & Material & Diâmetro & Peso linear & Comprimento & Rigidez & Coeficiente & T. Ruptura \\
\cline { 3 - 5 } & & $(\mathrm{mm})$ & $(\mathrm{t} / \mathrm{m})$ & $(\mathrm{m})$ & $(\mathrm{t})$ & de atrito & $(\mathrm{t})$ \\
\hline 1 & Amarra Grau 4 & 76.0 & 10,77 & 100.0 & 37225,00 & 1.000 & 600,1 \\
\hline 2 & Amarra Grau 4 & 105.0 & 20,55 & 1000.0 & 71046,80 & 1.000 & 1075,4 \\
\hline
\end{tabular}

\begin{tabular}{|c|c|}
\hline \multicolumn{2}{|c|}{ Âncora } \\
\hline Tipo & Stevpris MK III \\
\hline Poder de garra & $341,3(t)$ \\
\hline Peso & $15,0(t)$ \\
\hline
\end{tabular}

Tabela T3 - Resultados da utilização do algoritmo de otimização

\begin{tabular}{|r|r|r|r|r|r|r|r|r|r|r|}
\hline \multicolumn{4}{|c|}{ NAVIO } & \multicolumn{4}{|c|}{ REBOCADOR } & \multicolumn{2}{c|}{$\begin{array}{l}\text { Distância } \\
\text { Nav-Reb. }\end{array}$} & \multicolumn{2}{c|}{ Coord. da âncora } \\
\cline { 1 - 6 } Compr. & Tração & Ângulo & Tr. Horiz. & Compr. & Tração & Ângulo & Tr. Horiz. & $\mathbf{z}$ \\
\hline$(m)$ & $(t f)$ & $\left({ }^{\circ}\right)$ & $(t f)$ & $(m)$ & $(t f)$ & $\left({ }^{\circ}\right)$ & $(t f)$ & $(m)$ & $(m)$ & $(m)$ \\
\hline 174.5 & 27.21 & 0.15 & 2.93 & 79.1 & 26.89 & -0.15 & 2.93 & 76.6 & 67.7 & 78.7 \\
\hline 349.1 & 53.95 & 0.15 & 5.87 & 158.1 & 38.69 & -0.14 & 5.87 & 160.4 & 135.4 & 156.5 \\
\hline 523.6 & 59.47 & 0.15 & 8.80 & 237.2 & 33.71 & -0.14 & 6.41 & 572.9 & 524.5 & 232.6 \\
\hline 698.1 & 53.34 & 0.14 & 11.73 & 316.2 & 27.64 & -0.12 & 10.96 & 811.1 & 671.3 & 284.0 \\
\hline
\end{tabular}

\section{Fatores não presentes no modelo}

A finalidade do modelo aqui apresentado é o de acelerar o processo de cálculo para o planejamento da instalação de âncoras para equipamentos offshore. As principais restrições deste modelo em relação à situação real são as condições ambientais e a composição do leito oceânico.

As condições ambientais, como a presença de ondas, ventos e correntezas podem desviar a situação de cálculo da de instalação. 
Na presença de ondas tanto a embarcação de apoio quanto a embarcação que está sendo ancorada, e também a superfície do mar, não estarão estáticas e sim apresentando movimentos aleatórios.

No modelo apresentado, supõe-se que as embarcações estejam estáticas o que não corresponde à realidade. Porém, alguns fatores amenizam esta situação uma vez que a embarcação de apoio deve manter posição com auxílio dos propulsores controlados monitorados por posicionamento via satélite, GPS e a embarcação a ser ancorada ou é mantida na posição pelas linhas de ancoragem já instaladas ou por meio de outras embarcações de apoio utilizadas para reboque. No entanto o movimento vertical desta não se mantém sob controle.

Na figura 9 abaixo são mostradas as curvas de resposta de heave (movimento vertical) do navio utilizado como exemplo, em função da direção da onda incidente e da freqüência da onda. Tais respostas são obtidas para ondas de altura unitária.
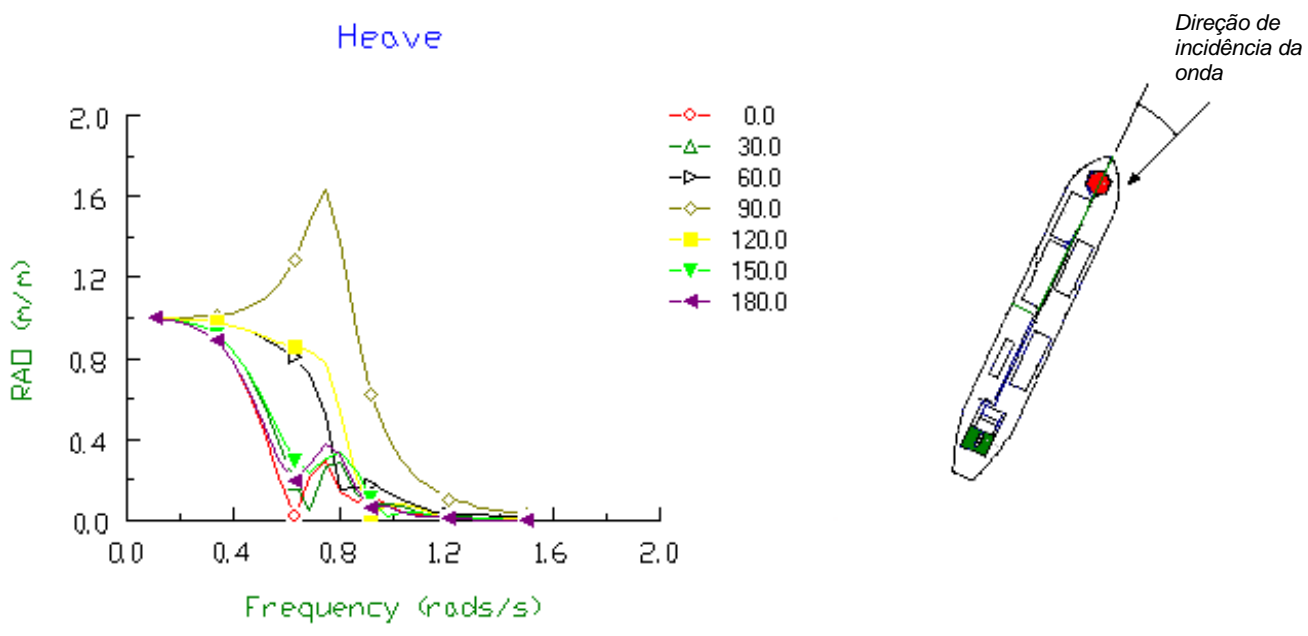

Figura 9 - Resposta em Heave para a embarcação ensaiada

Pode-se constatar que a máxima resposta ocorre para incidências de través (90 graus) e em freqüências em torno de $0.8 \mathrm{rads} / \mathrm{s}$ ou períodos da ordem de $8 \mathrm{~s}$. O valor de pico é de aproximadamente 1.6 metros para uma onda incidente de 1 metro de altura.

Na Tabela T4 são apresentadas as características de mar da locação onde foi instalada a embarcação do exemplo. Os valores, de altura máxima, altura significativa, possuem probabilidade de excedência de $1 \%$. Tais dados devem ser interpretados como sendo, para um período de (retorno) 1 ano a máxima altura de onda verificada, $11.6 \mathrm{~m}$, ocorre para as direções Sul, Sudoeste e Sudeste. A média do terço superior de todas as alturas vindas destas direções, Altura Significativa, é de $6.2 \mathrm{~m}$.

Outro dado importante é o período entre zeros e o período de pico, note-se que os valores se encontram na faixa entre 7 a 11s. Como o período em heave da embarcação é da ordem de $8 \mathrm{~s}$ há probabilidade de ressonância com amplificação do movimento de heave. 
Tabela T4 - Propriedades oceanográficas do local de instalação do navio do exemplo

\begin{tabular}{|c|c|c|c|c|c|c|c|}
\hline \multirow[b]{2}{*}{ DIRECTION } & \multirow[b]{2}{*}{ PARAMETER } & \multicolumn{6}{|c|}{ RETURN PERIOD (YEARS) } \\
\hline & & 1 & 10 & 20 & 30 & 50 & 100 \\
\hline $\mathrm{N}$ & $\begin{array}{r}\text { Hs } \\
\text { Tp } \\
\text { Hmax } \\
\text { THmax }\end{array}$ & $\begin{array}{c}3,0 \mathrm{~m} \\
8,9 \mathrm{~s} \\
5,6 \mathrm{~m} \\
8,3 \mathrm{~s}\end{array}$ & $\begin{array}{r}3,4 \mathrm{~m} \\
9,3 \mathrm{~s} \\
6,3 \mathrm{~m} \\
8,6 \mathrm{~s}\end{array}$ & $\begin{array}{r}3,7 \mathrm{~m} \\
9,6 \mathrm{~s} \\
6,9 \mathrm{~m} \\
8,9 \mathrm{~s}\end{array}$ & $\begin{array}{r}3,7 \mathrm{~m} \\
9,7 \mathrm{~s} \\
6,9 \mathrm{~m} \\
9,0 \mathrm{~s}\end{array}$ & $\begin{array}{r}4,1 \mathrm{~m} \\
9,8 \mathrm{~s} \\
7,6 \mathrm{~m} \\
9,1 \mathrm{~s}\end{array}$ & $\begin{array}{r}4,4 \mathrm{~m} \\
10,0 \mathrm{~s} \\
8,2 \mathrm{~m} \\
9,3 \mathrm{~s}\end{array}$ \\
\hline NE & $\begin{array}{r}\text { Hs } \\
\text { Tp } \\
\text { Hmax } \\
\text { THmax }\end{array}$ & $\begin{array}{c}3,6 \mathrm{~m} \\
10,0 \mathrm{~s} \\
6,7 \mathrm{~m} \\
9,3 \mathrm{~s}\end{array}$ & $\begin{array}{c}4,4 \mathrm{~m} \\
11,0 \mathrm{~s} \\
8,2 \mathrm{~m} \\
10,2 \mathrm{~s}\end{array}$ & $\begin{array}{r}5,3 \mathrm{~m} \\
12,2 \mathrm{~s} \\
9,9 \mathrm{~m} \\
11,3 \mathrm{~s}\end{array}$ & $\begin{array}{r}5,6 \mathrm{~m} \\
12,4 \mathrm{~s} \\
10,4 \mathrm{~m} \\
11,5 \mathrm{~s}\end{array}$ & $\begin{array}{r}6,0 \mathrm{~m} \\
12,7 \mathrm{~s} \\
11,2 \mathrm{~m} \\
11,8 \mathrm{~s}\end{array}$ & $\begin{array}{r}6,3 \mathrm{~m} \\
12,9 \mathrm{~s} \\
11,7 \mathrm{~m} \\
12,0 \mathrm{~s}\end{array}$ \\
\hline$E$ & $\begin{array}{r}\text { Hs } \\
T p \\
\text { Hmax } \\
\text { THmax }\end{array}$ & $\begin{array}{c}3,5 \mathrm{~m} \\
10,0 \mathrm{~s} \\
6,5 \mathrm{~m} \\
9,3 \mathrm{~s}\end{array}$ & $\begin{array}{c}4,5 \mathrm{~m} \\
11,2 \mathrm{~s} \\
8,4 \mathrm{~m} \\
10,4 \mathrm{~s}\end{array}$ & $\begin{array}{r}5,4 \mathrm{~m} \\
12,3 \mathrm{~s} \\
10,0 \mathrm{~m} \\
11,4 \mathrm{~s}\end{array}$ & $\begin{array}{r}5,9 \mathrm{~m} \\
12,8 \mathrm{~s} \\
11,0 \mathrm{~m} \\
11,9 \mathrm{~s}\end{array}$ & $\begin{array}{r}6,3 \mathrm{~m} \\
13,0 \mathrm{~s} \\
11,7 \mathrm{~m} \\
12,1 \mathrm{~s}\end{array}$ & $\begin{array}{r}6,5 \mathrm{~m} \\
13,0 \mathrm{~s} \\
12,1 \mathrm{~m} \\
12,1 \mathrm{~s}\end{array}$ \\
\hline SE, S e SW & $\begin{array}{r}\text { Hs } \\
T p \\
\text { Hmax } \\
\text { THmax }\end{array}$ & $\begin{array}{r}6,2 \mathrm{~m} \\
11,6 \mathrm{~s} \\
11,5 \mathrm{~m} \\
10,8 \mathrm{~s}\end{array}$ & $\begin{array}{r}7,8 \mathrm{~m} \\
12,3 \mathrm{~s} \\
14,5 \mathrm{~m} \\
11,4 \mathrm{~s}\end{array}$ & $\begin{array}{r}8,2 \mathrm{~m} \\
12,4 \mathrm{~s} \\
15,3 \mathrm{~m} \\
11,5 \mathrm{~s}\end{array}$ & $\begin{array}{r}8,4 \mathrm{~m} \\
12,5 \mathrm{~s} \\
15,6 \mathrm{~m} \\
11,6 \mathrm{~s}\end{array}$ & $\begin{array}{r}8,7 \mathrm{~m} \\
12,6 \mathrm{~s} \\
16,2 \mathrm{~m} \\
11,7 \mathrm{~s}\end{array}$ & $\begin{array}{r}9,1 \mathrm{~m} \\
12,8 \mathrm{~s} \\
16,9 \mathrm{~m} \\
11,9 \mathrm{~s}\end{array}$ \\
\hline W e NW & $\begin{array}{r}\text { Hs } \\
T p \\
\text { Hmax } \\
\text { THmax }\end{array}$ & $\begin{array}{c}2,7 \mathrm{~m} \\
8,3 \mathrm{~s} \\
5,0 \mathrm{~m} \\
7,7 \mathrm{~s}\end{array}$ & $\begin{array}{r}3,0 \mathrm{~m} \\
8,7 \mathrm{~s} \\
5,6 \mathrm{~m} \\
8,1 \mathrm{~s}\end{array}$ & $\begin{array}{r}3,4 \mathrm{~m} \\
8,9 \mathrm{~s} \\
6,3 \mathrm{~m} \\
8,3 \mathrm{~s}\end{array}$ & $\begin{array}{r}3,5 \mathrm{~m} \\
9,0 \mathrm{~s} \\
6,5 \mathrm{~m} \\
8,4 \mathrm{~s}\end{array}$ & $\begin{array}{r}3,7 \mathrm{~m} \\
9,2 \mathrm{~s} \\
6,9 \mathrm{~m} \\
8,5 \mathrm{~s}\end{array}$ & $\begin{array}{r}3,8 \mathrm{~m} \\
9,3 \mathrm{~s} \\
7,1 \mathrm{~m} \\
8,6 \mathrm{~s}\end{array}$ \\
\hline
\end{tabular}

Fazendo um pequeno exercício, deslocando-se o fair lead (topo da linha) de $\pm 0.5 *$ (6.2 $\mathrm{m}$ de onda x 1.2 resposta de pico em heave) $= \pm 3.2 \mathrm{~m}$ e comparando os resultados com os resultados desconsiderando tal movimento, conforme se mostra na figura 10, pode-se notar que as diferenças não são relevantes, corroborando a adequação da hipótese adotada no modelo de que ambas embarcações não apresentam movimentos durante a instalação.

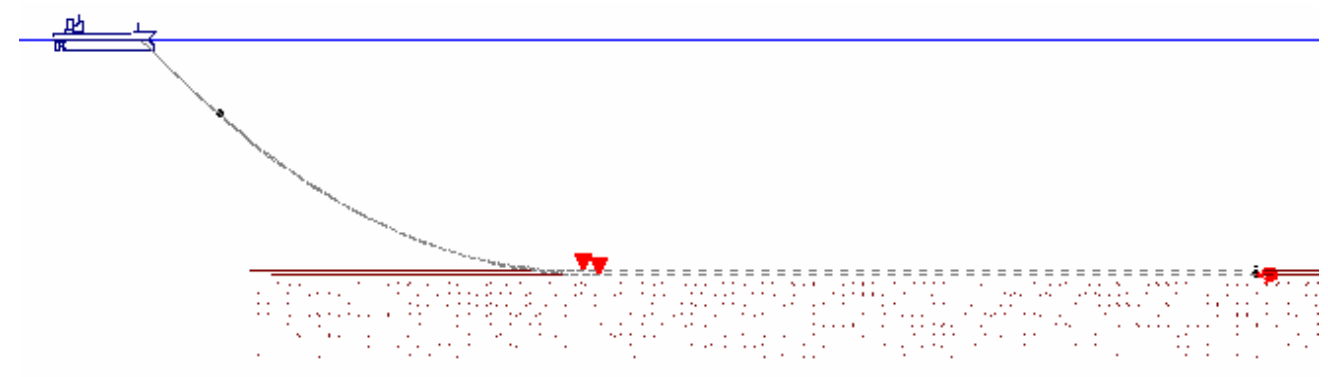

Figura 10 - Comparação dos resultados de configuração de linha com movimento extremo de heave no fair lead

Mesmo assim, as condições de onda aqui mostradas são de extremos que estariam ocorrendo na pior tempestade já observada no período de um ano, situação para a qual as operações de lançamento são proibitivas por motivos óbvios de segurança. Normalmente tais operações são realizadas com condições climáticas favoráveis, podendo-se aguardar até que elas aconteçam para se realizar a ancoragem. 
Os ventos, não caracterizam problemas para a linha e sim para as embarcações que, expostas ao vento sofrem forças proporcionais às suas áreas vélicas que tendem a afastá-las da posição. Neste caso, de modo semelhante ao que acontece com a deriva das embarcações quando expostas a ondas, os rebocadores de apoio procuram manter a embarcação a ser ancorada na posição e o rebocador de lançamento, por atuação nos propulsores, também se mantém posicionado.

Na região onde foi ancorada a embarcação, para um período de retorno de 1 ano, a velocidade de corrente máxima observada na superfície, é da $1.6 \mathrm{~m} / \mathrm{s}$. Na ancoragem do navio tomado como exemplo foram utilizadas amarras de $76 \mathrm{~mm}$ no topo, por questões operacionais de manuseio, e $105 \mathrm{~mm}$ no restante da linha, com um peso linear de $10.7 \mathrm{t} / \mathrm{m}$ e $20.7 \mathrm{t} / \mathrm{m}$, respectivamente. Utilizando a força de arrasto por unidade de comprimento segundo o modelo de Morrison, dada por

$$
f_{a}=\frac{1}{2} \rho c_{D} D v^{2}
$$

onde:

$f_{a}$ é a força por unidade de comprimento

$\rho$ é a densidade da água do mar

$c_{D}$ é o coeficiente de arrasto

$D$ é o diâmetro do segmento

$v$ é a velocidade do fluido normal ao corpo

com os valores $c_{D}=3$, comumente utilizado para amarras, v=1.6, $\rho=1.025$ e $\mathrm{D}=0.105 \mathrm{~m}$ obter-se-á uma força linear de $0.4 \mathrm{t} / \mathrm{m}$. Tal valor é cerca de $2 \%$ do valor devido à ação da gravidade e ocorrerá em condição extrema de correnteza.

Nota-se que tal força é muito inferior à força peso e, portanto, sua influência pode ser considerada desprezível na mudança de configuração da catenária calculada considerando-se somente o efeito da gravidade.

\section{Conclusões}

No trabalho, apresentou-se uma metodologia para a otimização dos procedimentos de instalação de âncoras de arrasto para equipamentos oceânicos utilizados na exploração e produção de petróleo no oceano.

Como variáveis de decisão a serem estabelecidas pelo corpo técnico responsável pela instalação da âncora procura-se minimizar o comprimento de work wire a ser lançado pela embarcação de apoio, sua força horizontal e ao mesmo tempo fazer com que a âncora atinja um alvo pré-determinado para a instalação.

Para trabalhar com esses objetivos distintos utilizou-se a técnica de otimização por múltiplos objetivos, onde a função de mérito, em função das variáveis de decisão, foi montada com desvios adimensionais dos objetivos anteriormente descritos. Para a solução do problema de minimização da função de mérito, utilizou-se, sem prejuízo de escolha de qualquer outro, um algoritmo de minimização seqüencial com penalizações exterior, para o trato de variáveis contínuas e discretas. 
O modelo adotado para o problema físico foi o clássico de catenária extensível que possui solução analítica para forças distribuídas em uma única direção somente. Tal modelo, apesar de possuir limitações, se mostrou adequado para o cálculo da ancoragem de embarcações na região da Bacia de Campos, onde embora a velocidade de correnteza seja considerada alta, esta não gera forças de correnteza a níveis que distorçam o modelo de cálculo apresentado.

Além de otimizar os recursos para a operação de instalação de âncoras, obtém-se, como resultado adicional do processo, a automação do cálculo de todas as etapas intermediarias de lançamento. Acredita-se que tal ferramenta se torne um poderoso aliado dos técnicos responsáveis pela ancoragem de equipamentos offshore para a indústria de produção de petróleo em águas profundas

\section{Referências Bibliográficas}

(1) Augusto, O.B. \& Kawano, A. (1998). A Mixed Continuous and Discrete Nonlinear Constrained Algorithm for Optimizing Ship Hull Structural Design. Ocean Engineering, an International Journal of Research and Development, Elsevier Science Ltd., 25(9), 793-811.

(2) Fu, J.; Fenton, R.G. \& Cleghorn, W.L. (1991). A Mixed Integer-Discrete-Continuous Programming Method and its Application to Engineering Design Optimization. Engineering Optimization, 17, 263-280.

(3) Gill, P.E.; Murray, W. \& Wright, M.H. (1981). Practical Optimization. Academic Press Limited.

(4) Novaes, A.N. (1978). Métodos de Otimização, Aplicação aos Transportes. Editora Edgar Blücher Ltda.

(5) Oppenheim, B.W. \& Wilson, P.A. (1982). Static 2-D solution of a mooring line of arbitrary composition in the vertical and horizontal operating modes. International Shipbuilding Progress, 29(334), 142-153, June.

(6) Parsons, M.G. (1975). Optimization Methods for Use in Computer-Aided Ship Design. The Society of Naval Architects and Marine Engineers First Ship Technology and Research (STAR) Symposium, Washington, D.C. August. 